Annals of Pure and Applied Mathematics

Vol. 17, No. 2, 2018, 221-232

ISSN: 2279-087X (P), 2279-0888(online)

Published on 18 June 2018

www.researchmathsci.org

DOI: http://dx.doi.org/10.22457/apam.v17n2a8

Annals of

Pure and Applied

Mathematics

\title{
Periodic Solutions of Gurtin-MacCamy Model and Extended Gurtin-MacCamy Model
}

\author{
Nazia Afrin ${ }^{1}$ and Md. Shahidul Islam ${ }^{2}$ \\ ${ }^{1}$ Department of Mathematics and Physical Sciences, East West University \\ Bangladesh. Email: n.afrin@ewubd.edu \\ ${ }^{2}$ Department of Mathematics, University of Dhaka \\ Bangladesh. Email: mshahidul11@yahoo.com
}

Received 16 April 2018; accepted 26 May 2018

\begin{abstract}
In this paper, we attempt to bring the basic concept of Gurtin-MacCamy population model. We have discussed extended Gurtin-MacCamy model which is an extension of the renowned Gurtin-MacCamy model. We discussed the periodic solutions of those population models.
\end{abstract}

Keywords: Gurtin-MacCamy model, resolvent kernel, Extended Gurtin-MacCamy model, Volterra Integral Equation

AMS Mathematics Subject Classification (2010): 45E10

\section{Introduction}

In different branches of science, mathematics is often use as a tool to understand the static and dynamical behavior of the system. Each emerging field of sciences, with their own set of constraints, offers mathematician a way to understand the systematic behavior of the mathematical equation used to predict the dynamical behavior. Mathematics has always benefited from its involvement with emerging sciences. Each successive interaction revitalizes and enhances the field.

Biomedical science is clearly the premier science of the foreseeable future. With the example of how mathematics has benefited from and influenced physics, it is clear that mathematicians should become involved in the biosciences which are likely to be the most important and exciting scientific discoveries of all time.

The increasing study of realistic and practically useful mathematical models in population biology, whether we are dealing with a human population with or without its age distribution, population of an endangered species, bacterial or viral growth and so on, is a reflection of their use in helping to understand the dynamic processes involved and in making practical predictions [1].

Late 18th-century biologists began to develop techniques in population modelling in order to understand dynamics of growing and shrinking populations of living organisms. Thomas Malthus was one of the first to note that populations grew with a geometric pattern while contemplating the fate of humankind. One of the most basic and milestone models of population growth were the Logistic model of population 
Nazia Afrin and Md. Shahidul Islam

growth formulated by Pierre François Verhulst in 1838. The logistic model takes the shape of a sigmoid curve and describes the growth of a population as exponential, followed by a decrease in growth, and bound by a carrying capacity due to environmental pressures. Population modelling became of particular interest to biologists in the 20th century as pressure on limited means of sustenance due to increasing human populations in parts of Europe were noticed by biologist like Raymond Pearl. In 1921, Pearl invited physicist Lotka to assist him in his lab. Lotka developed paired differential equations that showed the effect of a parasite on its prey. Mathematician Vito Volterra equated the relationship between two species independent from Lotka. Together, Lotka and Volterra formed the Lotka-Volterra model for competition that applies the logistic equation to two species illustrating competition, predation, and parasitism interactions between species. There is an extensive literature for linear models of age-dependent populations, but the theories of nonlinear models are much more recent. The inclusion of nonlinearities in the equations of age-dependent population models increases considerably not only their mathematical difficulties, but also their reliability in the physical description of behaviours of a population. The purpose of this work is to describe a more general class of non-linear population models.

We study the growth (or decay) in the number of individuals of a particular species in a given region. Immigration and emigration are assumed to play no significant role in the dynamics of the populations, and our model does not take into account the sex of the individuals. The purpose of this paper is to show the existence of periodic solution of the population models. To prove the existence of periodic solution of GurtinMacCamy model we consider the Volterra integral equation (VIE) [2] and compare the models equation with this and try to prove some assumptions.

The stability of the Gurtin-MacCamy's age-structured population dynamics model is investigated in [3]. The existence of asymptotically periodic solutions of a nonlinear Volterra integral equation is discussed in [4] and periodic and asymptotically periodic solutions of a Volterra integral equations is discussed in [5].

The paper is organized as follows. In section 2, some basic mathematical tools are discussed. In section 3, model is defined and in section 4, periodic solution of the models are discussed. Section 5 contains conclusions.

\section{Some mathematical tools}

\subsection{Prefatory on integral equation}

Many problems of mechanics, mathematical physics, modeling and technology lead to consideration of an equation of the form

$$
\varphi(x)-\lambda \int_{a}^{b} k(x, s) \varphi(s) d s=f(x)
$$

where, $\varphi(x)$ is an unknown function. These equations are known "integral equations," since the unknown function appears in them under the integral sign [6].

Here, $K(x, s)$ is called kernel, $f(x)$ is called free term or the right-hand side and lambda $(\lambda)$ is called the parameter of the equation.

There are basically two main classes known as Fredholm and Volterra equations. Fredholm equations involve definite integrals, while Volterra equations have the independent variable as one of the limits. Each of them can be subdivided as: 
Periodic Solutions of Gurtin-MacCamy Model and Extended Gurtin-MacCamy Model

$$
\begin{aligned}
& \text { Fredholm Type } 1 f(x)=\int_{a}^{b} k(x, s) \varphi(s) d s \\
& \text { Fredholm Type } 2 \quad \varphi(x)=\lambda \int_{a}^{b} k(x, s) \varphi(s) d s+f(x)
\end{aligned}
$$

and

$$
\text { Volterra Type } 1 f(x)=\int_{a}^{x} k(x, s) \varphi(s) d s
$$

Volterra Type $2 \quad \varphi(x)=\lambda \int_{a}^{x} k(x, s) \varphi(s) d s+f(x)$

Integral equations are often easier to solve than a corresponding differential equation. One of the reasons is that the truncation errors of the solution tend to be averaged out by the process of quadrature while they tend to accumulate during the process of numerical integration employed in the solution of differential equations.

\subsection{Resolvent kernel}

Let $r(x, s ; \lambda)$ is the resolvent kernel of Volterra Integral Equation. The process of determining resolvent kernel and using the resolvent kernel the solution of the equation can be obtained in [7].

\subsection{Fixed point theorems}

Fixed point theorems are the most important tools in analysis for proving the existence and uniqueness of solutions to some problems.

Theorem 2.3.1. (Contraction mapping principle)

Let $(X, d)$ be a complete metric space and $F: X \rightarrow X$ be a contraction mapping, that is, there is $0<k<1$ such that $\forall x_{1}, x_{2} \in X$, we have $d\left(F x_{1}, F x_{2}\right) \leq k \cdot d\left(x_{1}, x_{2}\right)$.

Then there exists a unique fixed point of $F$ in $X$ and the orbit of any point is forward asymptotic to that fixed point.

The proof of this theorem can be found in any good introductory text on metric spaces and is based on an iterative method (see Smart [8, Theorem 1.2.2]).

Definition 2.1. Let $X$ and $Y$ be normed spaces. An operator $T: X \rightarrow Y$ is called compact if the image $T(M)$ of every bounded subset $M$ of $X$ is a relatively compact subset of $Y$, that is, its closure $T(M)$ is a compact subset of $Y$.

Theorem 2.3.2. (Schauder-Tychonov theorem)

Let $E$ be a non-empty closed, convex subset of a complete metric space $X$. Then every continuous map of $E$ into itself has at least one fixed point. Before using SchauderTychonov fixed point theorem, we would like to remind about Arzel'a-Ascoli theorem. For this, we first need to define the concept of equicontinuity of a family of maps under the supremum norm.

Definition 2.2. A family $F$ of functions on a metric space $(X, d)$ is equicontinuous if for every $\epsilon>0$, there exists $\delta>0$ such that if $x, y \in X$ and $d(x, y)<\delta$, then $\mid f(x)-$ $f(y) \mid<\epsilon$ for all $f \epsilon F$.

Theorem 2.3.3. (Arzel’a-Ascoli theorem) 
Nazia Afrin and Md. Shahidul Islam

Let $(X, d)$ be a compact metric space and let $C(X)$ denote the set of all continuous functions on $X$. A closed subspace of $C(X)$ is compact if and only if it is uniformly bounded and equicontinuous.

\subsection{Integral equations}

We now established an important formula by integrating the following partial differential equation along characteristics.

We denote the derivative by $D_{e}$ in the direction of the vector $e=(1,1) \in \mathbb{R}^{2}$, that is,

$$
D_{e} x(t, a)=\lim _{h \rightarrow 0} \frac{x(t+h, a+h)-x(t, a)}{h}
$$

Lemma 2.4.1. Let $p: \mathbb{R} \rightarrow \mathbb{R}_{+}$be a fixed measurable function and $\varphi \in L_{1}^{+}\left(\mathbb{R}_{+}\right)$. Then there is a unique solution $x: \mathbb{R} \times \mathbb{R}_{+} \rightarrow \mathbb{R}_{+}$to the partial differential equation (PDF)

$$
D_{e} x(t, a)=-\mu(t, a, p(t)) x(t, a), a>0, t \in \mathbb{R}
$$

with initial condition $\quad x(0, a)=\varphi(a), a \geq 0$

(2)

To prove this first we need to convert the system (1) and (2) in to an equivalent system of an integral equation (see Corduneanu[9, $\xi$ 3.1]). Let $x: \mathbb{R} \times \mathbb{R}_{+} \rightarrow \mathbb{R}_{+}$be a function and choose a pair of arbitrary, but fixed, numbers $\left(t_{0}, a_{0}\right) \in \mathbb{R} \times \mathbb{R}_{+}$, and denote

$$
\begin{gathered}
\bar{x}(h)=x\left(t_{0}+h, a_{0}+h\right), \\
\bar{\mu}(h)=\mu\left(t_{0}+h, a_{0}+h, p\left(t_{0}+h\right)\right) .
\end{gathered}
$$

Then equation (1) can be written as

$$
\frac{d \bar{x}}{d h}=-\bar{\mu}(h) \bar{x}(h)
$$

This equation has the following solution:

$$
\bar{x}(h)=x\left(t_{0}, a_{0}\right) \exp \left\{-\int_{0}^{h} \bar{\mu}(\tau) d \tau\right\}
$$

This relation gives the values of $x$ at all points on the characteristic through $\left(t_{0}, a_{0}\right)$.

In particular, if we take $a_{0}=a-t, t_{0}=0$ and $h=t$, using condition (2), we obtain,

$$
x(t, a)=\varphi(a-t) \exp \left\{-\int_{0}^{t} \mu(\tau, a+\tau-t, p(\tau)) d \tau\right\}
$$

(4) is the unique solution of the differential equation (1) with initial condition (2).

\section{The model}

\subsection{Non-linear age-time dependent Gurtin-Maccamy model (GMC Model)}

Lotka's and Leslies's models cannot be used for long term projections since, just Malthus's model; they predict population explosion or extinction. The main reason for this type of long time behavior is that all these models are represented by linear equations. In contrast, Verhulst's model-created to remedy the inability of Malthus's to capture the long-term behaviour of natural population is nonlinear. It is generated by assuming that the growth rate of population depends on the total population. In particular this means that the per capita birth rate and the per capita death rate are assumed to 
Periodic Solutions of Gurtin-MacCamy Model and Extended Gurtin-MacCamy Model

depend on the total population. Following this idea Morton Gurtin and Richard MacCamy [10] modified the McKendrick-Vonforester model so that the per capita age structured birth rate and the per capita age structured death rate depend on the total population.

In this paper, we consider a model based on the paper [11] in the age-time continuum in which the dependence of natality and mortality rates on age, time and population size incorporated. Thus, the model under consideration is an extension of the non-linear model of Gurtin and MacCamy.

The Gurtin-MacCamy's age-structured population dynamics model has been studied in Gurtin, et al. (1974). The model is based on the following initial value problem:

$$
\begin{gathered}
D x(a, t)+\mu(a, P(t)) x(a, t)=0, \quad a>0, \quad t>0 \\
x(0, t)=\int_{0}^{\infty} v(a, P(t)) x(a, t) d a, \quad t>0 \\
x(a, 0)=\varphi(a), a \geq 0
\end{gathered}
$$

where, $P(t)=\int_{0}^{\infty} x(a, t) d a, t>0$

The parameter $\varphi$ is the initial age distribution, $u$ is the mortality function and $v$ is the natality function, where, $x(a, t)$ is the density of the population with respect to the chronological age $a[0, \infty)$ at time $t \geq 0 ; P(t)=\int_{0}^{\infty} x(a, t) d a$ is the total population size at time $t ; v(a, P(t)) ; \mu(a, P(t))$ are respectively, the birth rate i.e. the average number of offspring per unit time, produced by an individual of age $a$ when the population size is $P(t)$, and the mortality rate i.e. the death rate at age $a$, per unit population when the population size is $P(t)$;

$$
x(0, t)=\int_{0}^{\infty} v(a, P(t)) x(a, t) d a
$$

is the number of births per unit time, when the population size is $P(t)$.

For Gurtin-MacCamy model birth rate of a population is not defined for past but the model has a unique solution in the past under certain additional conditions on the birth rate of the population.

We can write the above initial value problem as

$$
\begin{gathered}
\frac{\partial x(a, t)}{\partial a}+\frac{\partial x(a, t)}{\partial t}+\mu(a, P(t)) x(a, t)=0, \quad t>0, a>0 \\
x(0, t)=\int_{0}^{\infty} v(a, P(t)) x(a, t) d a, \quad t>0 \\
x(a, 0)=\varphi(a), \quad a \geq 0
\end{gathered}
$$

where, $P(t)=\int_{0}^{\infty} x(a, t) d a, t>0$ the parameters $\varphi, \mu$ and $v$ being the same as before.

\section{The model: Basic equations}

$$
P(t)=\int_{0}^{t} B(a-t) \Pi(a, t ; P) d a+\int_{t}^{\infty} \varphi(a-t) \lambda(a, t ; P) d a
$$


Nazia Afrin and Md. Shahidul Islam

(5) $B(t)=\int_{0}^{t} v(a, t ; P(t)) B(t-a) \Pi(a, t ; P) d a+\int_{t}^{\infty} v(a, t ; P(t)) \varphi(a-$ t) $\lambda(a, t ; P) d a=\int_{0}^{t} v(t-a, t ; P(t)) \Lambda(a, t ; P) B(a) d a+\int_{0}^{\infty} v(t+$ $a, t ; P(t)) \Gamma(a, t ; P) \varphi(a) d a$

where, $\Lambda(a, t ; P)=\Pi(t-a, t ; P)=\exp \left\{-\int_{a}^{t} \mu(\tau-a, \tau, P(\tau)) d \tau\right\}$ and, $\Gamma(a, t ; P)=\lambda(t+a, t ; P)=\exp \left\{-\int_{0}^{t} \mu(\tau+a, \tau, P(\tau)) d \tau\right\}$.

This is the required non-linear integral equation.

In the paper [12], Existence of the solution of the integral equation has been discussed on the basis of Banach's Fixed Point theorem.

\subsection{Extended Gurtin-Maccamy model (EGMC Model)}

To overcome the deficiency of Sharpe-Lotka and Gurtin-MacCamy models, we study different models in which natality and mortality moduli are simultaneously age, time and population dependent. Nonlinear age-time dependent models of population provide a biologically more realistic description of the behaviour of the population when the past history of the population is considered. The problem can be stated as the following initial-boundary value problem:

$$
\begin{gathered}
D x(t, a)+\mu(t, a, P(t)) x(t, a)=0, \quad a>0, t \in \mathbb{R} \\
x(t, 0)=\int_{0}^{\infty} v(t, a, P(t)) x(t, a) d a, \quad t \in \mathbb{R} \\
x(0, a)=\varphi(a), \quad a \geq 0
\end{gathered}
$$

the parameters $\varphi, \mu$ and $\nu$ being the same as before.

Extended Gurtin-MacCamy model solution for $(\mu, v) \in \boldsymbol{M} \times \boldsymbol{N}$ is a pair $(B, P) \in C^{2}$ satisfying the equations

$$
B(t)=\int_{-\infty}^{t} v(t, t-s, P(t)) \exp \left\{-\int_{s}^{t} \mu(\tau, \tau-s, P(\tau)) d \tau\right\} B(s) d s
$$

$$
P(t)=\int_{-\infty}^{t} \exp \left\{-\int_{s}^{t} \mu(\tau, \tau-s, P(\tau)) d \tau\right\} B(s) d s
$$

where $t \in \mathbb{R}$.

\section{Periodic solution of GMC and EGMC model}

We consider the integral equation

$$
x(t)=f(t)+\int_{-\infty}^{t} K(t, s) x(s) d s,-\infty<t<\infty
$$

and compare the Gurtin-MacCamy model and Extended Gurtin-MacCamy model equation with (9).

We discuss the periodic solution of the model equation by using the resolvent kernel and the contraction mapping principle.

Periodic solution using resolvent kernel

From equation (5) and (6), we have 
Periodic Solutions of Gurtin-MacCamy Model and Extended Gurtin-MacCamy Model $B(t)=\int_{0}^{t} v(t-a, t, P(t)) \exp \left\{-\int_{a}^{t} \mu(\tau-a, \tau, P(\tau)) d \tau\right\} B(a) d a$
$+\int_{0}^{\infty} v\left((t a, t, P(t)) \exp \left\{-\int_{0}^{t} \mu(\tau+\right.\right.$

$a, \quad \tau, P(\tau)) d \tau\} \varphi(a) d a$

$P(t)=\int_{0}^{t} \exp \left\{-\int_{a}^{t} \mu(\tau-a, \tau, P(\tau)) d \tau\right\} B(a) d a+\int_{0}^{\infty} \exp \left\{-\int_{0}^{t} \mu(\tau+\right.$ $a, \tau, P(\tau)) d \tau\} \varphi(a) d a$

Lemma 4.1. If $P(t)$ is a solution of (11) then $P(t+T)$ is also a solution of (11).

To prove this, we need to find its solution by using resolvent kernel for both time at $t$ and $t+T$. Then we will take the difference between them which is very small (say $\epsilon$ )

Proof: We have

From (11), we get

$$
B=\zeta P
$$

$P(t)=\int_{0}^{t} \exp \left\{-\int_{a}^{t} \mu(\tau-a, \tau, P(\tau)) d \tau\right\} \zeta P(a) d a+\int_{0}^{\infty} \exp \left\{-\int_{0}^{t} \mu(\tau+\right.$ $a, \tau, P(\tau)) d \tau\} \varphi(a) d a$

which is Volterra Integral Equation (VIE) of second kind with $\lambda=1$.

Comparing (12) with VIE we get,

$$
f(t)=\int_{0}^{\infty} \exp \left\{-\int_{0}^{t} \mu(\tau+a, \tau, P(\tau)) d \tau\right\} \varphi(a) d a
$$

and

$$
K(t, a)=\zeta \exp \left\{-\int_{a}^{t} \mu(\tau-a, \tau, P(\tau)) d \tau\right\}
$$

We know that $K_{1}(t, a)=K(t, a), K_{n}(t, a)=\int_{a}^{t} K(t, u) K_{n-1}(u, a) d u$

Substituting $n=2,3,4 \ldots$ in the relation (14), we have

$$
\begin{aligned}
& K_{2}(t, a)=\int_{a}^{t} K(t, u) K_{1}(u, a) d u \\
& =\int_{a}^{t} \zeta \exp \left\{-\int_{u}^{t} \mu(\tau-a, \tau, P(\tau)) d \tau\right\} \quad \zeta \exp \left\{-\int_{a}^{u} \mu(\tau\right. \\
& \quad-a, \tau, P(\tau)) d \tau\} d u \\
& =\zeta^{2} \int_{a}^{t} \exp \left\{-\int_{a}^{t} \mu(\tau-a, \tau, P(\tau)) d \tau\right\} d u \\
& =\zeta^{2} \int_{a}^{t} \exp \{-\underline{\mu}(t-a)\} d u \\
& =\zeta^{2} \exp \{-\underline{\mu}(t-a)\}(t-a) \\
& \therefore K_{2}(t, a)=\zeta^{2} \exp \{-\underline{\mu}(t-a)\}(t-a) \\
& \text { Now, for } n=3, \quad K_{3}(t, a)=\zeta^{3} \exp \{-\underline{\mu}(t-a)\} \frac{(t-a)^{2}}{2 !} \\
& \text { Similarly, for } n=4, K_{4}(t, a)=\zeta^{4} \exp \{-\underline{\mu}(t-a)\} \frac{(t-a)^{3}}{3 !}
\end{aligned}
$$


Nazia Afrin and Md. Shahidul Islam

By mathematical induction we can show that

Resolvent kernel,

$$
K_{n}(t, a)=\zeta^{n} \exp \{-\underline{\mu}(t-a)\} \frac{(t-a)^{n-1}}{(n-1) !}
$$

$$
\begin{gathered}
r(t, a)=\sum_{n=1}^{\infty} K_{n}(t, a)=\sum_{n=1}^{\infty} \zeta^{n} \exp \{-\underline{\mu}(t-a)\} \frac{(t-a)^{n-1}}{(n-1) !} \\
=\sum_{n=1}^{\infty} \zeta \exp \{-\underline{\mu}(t-a)\} \frac{\{\zeta(t-a)\}^{n-1}}{(n-1) !} \\
=\zeta \exp \{-\underline{\mu}(t-a)\} \exp \{\zeta(t-a)\}
\end{gathered}
$$

The solution of equation (12) is,

$$
\begin{aligned}
& P(t)=f(t)+\int_{0}^{t} r(t, a) f(a) d a \\
& \quad=f(t)+\int_{0}^{t} \zeta \exp \{-\underline{\mu}(t-a)\} \exp \{\zeta(t-a)\} f(a) d a=I+I I
\end{aligned}
$$

$$
\begin{gathered}
I=\int_{0}^{\infty} \exp \left\{-\int_{0}^{t} \mu(\tau+a, \tau, P(\tau)) d \tau\right\} \varphi(a) d a=\int_{0}^{\infty} \exp (-\underline{\mu} t) \varphi(a) d a \\
\leq \int_{0}^{\infty} \varphi(a) d a[\exp (-\underline{\mu} t) \leq 1]=\phi \\
I I=\int_{0}^{t} \zeta \exp \{-\underline{\mu}(t-a)\} \exp \{\zeta(t-a)\} f(a) d a \\
=\int_{0}^{t} \zeta \exp \{-\underline{\mu}(t-a)\} \exp \{\zeta(t-a)\} \phi d a \\
\leq \int_{0}^{t} \zeta \exp \{\zeta(t-a)\} \phi d a
\end{gathered}
$$

From (15), we get

$$
P(t) \leq \phi+\int_{0}^{t} \zeta \exp \{\zeta(t-a)\} \phi d a
$$

From (11), we get for $t+T$

$$
\begin{aligned}
P(t+T)=\int_{0}^{t+T} & \exp \left\{-\int_{a}^{t+T} \mu(\tau-a, \tau, P(\tau)) d \tau\right\} \zeta P(a) d a \\
& +\int_{0}^{\infty} \exp \left\{-\int_{0}^{t+T} \mu(\tau+a, \tau, P(\tau)) d \tau\right\} \varphi(a) d a
\end{aligned}
$$

which is also Volterra Integral Equation (VIE) of second kind with $\lambda=1$.

Comparing (17) with VIE we get,

and

$$
f(t+T)=\int_{0}^{\infty} \exp \left\{-\int_{0}^{t+T} \mu(\tau+a, \tau, P(\tau)) d \tau\right\} \varphi(a) d a
$$

$$
K(t+T, a)=\zeta \exp \left\{-\int_{a}^{t+T} \mu(\tau-a, \tau, P(\tau)) d \tau\right\}
$$


Periodic Solutions of Gurtin-MacCamy Model and Extended Gurtin-MacCamy Model

We know that

$K_{1}(t+T, a)=K(t+T, a), K_{n}(t+T, a)=\int_{a}^{t+T} K(t+T, u) K_{n-1}(u, a) d u$ (19)

Substituting $n=2,3,4 \ldots$ in the relation (19), we have

$$
\begin{aligned}
& K_{2}(t+T, a)=\int_{a}^{t+T} K(t+T, u) K_{1}(u, a) d u \\
& =\int_{a}^{t+T} \zeta \exp \left\{-\int_{u}^{t+T} \mu(\tau-a, \tau, P(\tau)) d \tau\right\} . \quad \zeta \exp \left\{-\int_{a}^{u} \mu(\tau\right. \\
& \quad-a, \tau, P(\tau)) d \tau\} d u \\
& \quad=\zeta^{2} \int_{a}^{t+T} \exp \left\{-\int_{a}^{t+T} \mu(\tau-a, \tau, P(\tau)) d \tau\right\} d u \\
& \quad=\zeta^{2} \exp \{-\underline{\mu}(t+T-a)\}(t+T-a) \\
& \therefore K_{2}(t+T, a)=\zeta^{2} \exp \{-\underline{\mu}(t+T-a)\}(t+T-a)
\end{aligned}
$$

Now, for $n=3, \quad K_{3}(t+T, a)=\zeta^{3} \exp \{-\underline{\mu}(t+T-a)\} \frac{(t+T-a)^{2}}{2 !}$

Similarly, for $n=4, \quad K_{4}(t+T, a)=\zeta^{4} \exp \{-\underline{\mu}(t+T-a)\} \frac{(t+T-a)^{3}}{3 !}$

By mathematical induction we can show that

$$
K_{n}(t+T, a)=\zeta^{n} \exp \{-\underline{\mu}(t+T-a)\} \frac{(t+T-a)^{n-1}}{(n-1) !}
$$

Resolvent kernel,

$$
\begin{gathered}
r(t+T, a)=\sum_{n=1}^{\infty} K_{n}(t+T, a) \\
=\sum_{n=1}^{\infty} \zeta^{n} \exp \{-\underline{\mu}(t+T-a)\} \frac{(t+T-a)^{n-1}}{(n-1) !} \\
=\sum_{n=1}^{\infty} \zeta \exp \{-\underline{\mu}(t+T-a)\} \frac{\{\zeta(t+T-a)\}^{n-1}}{(n-1) !} \\
=\zeta \exp \{-\underline{\mu}(t+T-a)\} \exp \{\zeta(t+T-a)\}
\end{gathered}
$$

The solution of equation (17) is,$$
P(t+T)=f(t+T)+\int_{0}^{t+T} r(t+T, s) f(a) d a
$$$$
=f(t+T)+\int_{0}^{t+T} \zeta \exp \{-\underline{\mu}(t+T-a)\} \exp \{\zeta(t+T-a)\} f(a) d a=I I I+
$$$$
\text { IV }
$$

Here,

$$
I I I=\int_{0}^{\infty} \exp \left\{-\int_{0}^{t+T} \mu(\tau+a, \tau, P(\tau)) d \tau\right\} \varphi(a) d a
$$


Nazia Afrin and Md. Shahidul Islam

$$
\begin{gathered}
=\int_{0}^{\infty} \exp \{-\underline{\mu}(t+T)\} \varphi(a) d a \leq \int_{0}^{\infty} \varphi(a) d a[\exp \{-\underline{\mu}(t+T) \leq 1] \leq \phi \\
I V=\int_{0}^{t+T} \zeta \exp \{-\underline{\mu}(t+T-a)\} \exp \{\zeta(t+T-a)\} f(a) d a \\
=\int_{0}^{t+T} \zeta \exp \{-\underline{\mu}(t+T-a)\} \cdot \exp \{\zeta(t+T-a)\} \phi d a \\
\leq \int_{0}^{t+T} \zeta \exp \{\zeta(t+T-a)\} \phi d a
\end{gathered}
$$

From (20), we get

$$
P(t+T) \leq \phi+\int_{0}^{t+T} \zeta \exp \{\zeta(t+T-a)\} \phi d a
$$

Now,

$$
\begin{aligned}
& |P(t+T)-P(t)| \\
& \leq\left|\phi \zeta\left[\int_{0}^{t+T} \exp \{\zeta(t+T-a)\} d a-\int_{0}^{t} \exp \{\zeta(t-a)\} d a\right]\right| \\
& \leq\left|\phi \zeta\left[\frac{1-\exp \{\zeta(t+T)\}}{\zeta}-\frac{1-\exp \{\zeta t\}}{\zeta}\right]\right| \leq \phi|[\exp (\zeta t)-\exp \{\zeta(t+T)\}]| \\
& \leq \phi|\zeta t-\zeta t-\zeta T|\left[\left|e^{x}-e^{y}\right| \leq|x-y|\right]=\phi|-\zeta T|= \\
& \phi \zeta T \leq \epsilon\left[\phi \leq \frac{\epsilon}{\zeta T}\right] \\
& |P(t+T)-P(t)| \leq \epsilon
\end{aligned}
$$

We have

Then

$$
B(t)=\zeta P(t) \text { and } B(t+T)=\zeta P(t+T)
$$

$$
\begin{gathered}
|B(t+T)-B(t)|=|\zeta P(t+T)-\zeta P(t)| \leq \zeta|P(t+T)-P(t)| \leq \zeta \epsilon \leq \epsilon \\
|B(t+T)-B(t)| \leq \epsilon .
\end{gathered}
$$

Proof of Lemma 4.1 (For Extended Gurtin-MacCamy model)

From (8) we have

$$
\begin{aligned}
& P(t)=\int_{-=0}^{t} \exp \left\{-\int_{s}^{t} \mu(\tau, \tau-s, P(\tau)) d \tau\right] B(s) d s \\
& \quad=\int_{-\infty}^{0} \exp \left\{-\int_{s}^{t} \mu(\tau, \tau-s, P(\tau)) d \tau\right\} B(s) d s+\int_{0}^{t} \exp \left\{-\int_{s}^{t} \mu(\tau, \tau-\right. \\
& s, P(\tau)) d \tau\} B(s) d s
\end{aligned}
$$

We have

$$
B=\zeta P
$$


Periodic Solutions of Gurtin-MacCamy Model and Extended Gurtin-MacCamy Model

$\therefore P(t)=\int_{-\infty}^{0} \exp \left\{-\int_{s}^{t} \mu(\tau, \tau-s, P(\tau)) d \tau\right\} \zeta P(s) d s+\int_{0}^{t} \exp \left\{-\int_{s}^{t} \mu(\tau, \tau-\right.$ $s, P(\tau)) d \tau\} \zeta P(s) d s$

which is Volterra Integral Equation(VIE) of second kind with $\lambda=1$.

Comparing (22) with VIE we get,

$$
\begin{aligned}
f(t) & =\int_{-\infty}^{0} \exp \left\{-\int_{s}^{t} \mu(\tau, \tau-s, P(\tau)) d \tau\right\} \zeta P(s) d s \\
& \text { and } K(t, s)=\zeta \exp \left\{-\int_{s}^{t} \mu(\tau, \tau-s, P(\tau)) d \tau\right\}
\end{aligned}
$$

We know that

$$
K_{1}(t, s)=K(t, s) \text { and } \quad K_{n}(t, s)=\int_{s}^{t} K(t, u) K_{n-1}(u, s) d u
$$

Substituting $n=2,3,4 \ldots$ in the relation (24), we have

$$
K_{2}(t, s)=\int_{s}^{t} K(t, u) K_{1}(u, s) d u=\zeta^{2} \exp \{-\underline{\mu}(t-s)\}(t-s)
$$

Now, for $n=3, K_{3}(t, s)=\int_{s}^{t} K(t, u) K_{2}(u, s) d u=\zeta^{3} \exp \{-\underline{\mu}(t-s)\} \frac{(t-s)^{2}}{2 !}$

Similarly, for $n=4, \quad K_{4}(t, s)=\zeta^{4} \exp \{-\underline{\mu}(t-s)\} \frac{(t-s)^{3}}{3 !}$

By mathematical induction we can show that

Resolvent kernel,

$$
K_{n}(t, s)=\zeta^{n} \exp \{-\underline{\mu}(t-s)\} \frac{(t-s)^{n-1}}{(n-1) !}
$$

$$
\begin{array}{r}
r(t, s)=\sum_{n=1}^{\infty} K_{n}(t, s)=\sum_{n=1}^{\infty} \zeta^{n} \exp \{-\underline{\mu}(t-s)\} \frac{(t-s)^{n-1}}{(n-1) !} \\
=\sum_{n=1}^{\infty} \zeta \exp \{-\underline{\mu}(t-s)\} \frac{\{\zeta(t-s)\}^{n-1}}{(n-1) !} \\
=\zeta \exp \{-\underline{\mu}(t-s)\} \exp \{\zeta(t-s)\}
\end{array}
$$

The solution of equation (8) is,

$P(t)=f(t)+\int_{0}^{t} r(t, s) f(s) d s=f(t)+\int_{0}^{t} \zeta \exp \{-\underline{\mu}(t-s)\} \exp \{\zeta(t-$ $s)\} f(s) d s=I+I I$

Here,

$$
\begin{array}{r}
I=\int_{-\infty}^{0} \exp \left\{-\int_{s}^{t} \mu(\tau, \tau-s, P(\tau)) d \tau\right\} \zeta P(s) d s \\
=\int_{-\infty}^{0} \exp \{-\underline{\mu}(t-s)\} \zeta P(s) d s
\end{array}
$$


Nazia Afrin and Md. Shahidul Islam

$$
\begin{gathered}
\leq \int_{-\infty}^{0} \zeta P(s) d s[\exp \{-\underline{\mu}(t-s)\} \leq 1] \leq \int_{-\infty}^{0} B(s) d s \\
I I=\int_{0}^{t} \zeta \exp \{-\underline{\mu}(t-s)\} \exp \{\zeta(t-s)\} f(s) d s \\
=\int_{0}^{t}\left[\zeta \operatorname { e x p } \{ - \underline { \mu } ( t - s ) \} \operatorname { e x p } \{ \zeta ( t - s ) \} \left\{\int_{-\infty}^{0} \exp \{-\underline{\mu}(s\right.\right. \\
-u)\} \zeta P(u) d u\}] d s \\
\leq \int_{0}^{t} \zeta \exp \{\zeta(t-s)\}\left[\int_{-\infty}^{0} \zeta P(u) d u\right] d s \\
\leq \int_{0}^{t} \zeta \exp \{\zeta(t-s)\}\left[\int_{-\infty}^{0} B(u) d u\right] d s
\end{gathered}
$$

From (25)

$$
P(t) \leq \int_{-\infty}^{0} B(s) d s+\int_{0}^{t} \zeta \exp \{\zeta(t s)\}\left[\int_{-\infty}^{0} B(u) d u\right] d s
$$

Now, we will try to find the solution for $t+T$ then the equation (8) becomes

$$
\begin{gathered}
P(t+T)=\int_{-\infty}^{t+T} \exp \left\{-\int_{s}^{t+T} \mu(\tau, \tau-s, P(\tau)) d \tau\right\} B(s) d s \\
=\int_{-\infty}^{t+T} \exp \left\{-\int_{s}^{t+T} \mu(\tau, \tau-s, P(\tau)) d \tau\right\} \zeta P(s) d s \\
=\int_{-\infty}^{0} \exp \left\{-\int_{s}^{t+T} \mu(\tau, \tau-s, P(\tau)) d \tau\right\} \zeta P(s) d s \\
+\int_{0}^{t+T} \exp \left\{-\int_{s}^{t+T} \mu(\tau, \tau-s, P(\tau)) d \tau\right\} \zeta P(s) d s
\end{gathered}
$$

Again, comparing with VIE, we get

We know that

$$
\begin{gathered}
f(t+T)=\int_{-\infty}^{0} \exp \left\{-\int_{s}^{t+T} \mu(\tau, \tau-s, P(\tau)) d \tau\right\} \zeta P(s) d s \\
K(t+T, s)=\zeta \exp \left\{-\int_{s}^{t+T} \mu(\tau, \tau-s, P(\tau)) d \tau\right\}
\end{gathered}
$$

$K_{1}(t+T, s)=K(t+T, s)$ and $K_{n}(t+T, s)=\int_{s}^{t+T} K(t+T, u) K_{n-1}(u, s) d u$ (28)

Substituting $n=2,3,4 \ldots$ in (28), we get

$$
\begin{aligned}
K_{2}(t+T, s)= & \int_{s}^{t+T} K(t+T, u) K_{1}(u, s) d u \\
& =\zeta^{2} \exp \{-\underline{\mu}(t+T-s)\}(t+T-s)
\end{aligned}
$$

Now, for $n=3$,

$$
K_{3}(t+T, s)=\int_{s}^{t+T} K(t+T, u) K_{2}(u, s) d u=\zeta^{3} \exp \{-\underline{\mu}(t+T-s)\} \frac{(t+T-s)^{2}}{2 !}
$$


Periodic Solutions of Gurtin-MacCamy Model and Extended Gurtin-MacCamy Model

Similarly, for $n=4, K_{4}(t+T, s)=\zeta^{4} \exp \{-\underline{\mu}(t+T-s)\} \frac{(t+T-s)^{3}}{3 !}$

By mathematical induction we can show that

Resolvent kernel,

$$
K_{n}(t+T, s)=\zeta^{n} \exp \{-\underline{\mu}(t+T-s)\} \frac{(t+T-s)^{n-1}}{(n-1) !}
$$

$$
\begin{gathered}
r(t+T, s)=\sum_{n=1}^{\infty} K_{n}(t+T, s)=\sum_{n=1}^{\infty} \zeta^{n} \exp \{-\underline{\mu}(t+T-s)\} \frac{(t+T-s)^{n-1}}{(n-1) !} \\
=\sum_{n=1}^{\infty} \zeta \exp \{-\underline{\mu}(t+T-s)\} \frac{\{\zeta(t+T-s)\}^{n-1}}{(n-1) !} \\
=\zeta \exp \{-\underline{\mu}(t+T-s)\} \exp \{\zeta(t+T-s)\}
\end{gathered}
$$

The solution of equation (27) is,

$$
\begin{aligned}
& P(t+T)=f(t+T)+\int_{0}^{t+T} r(t+T, s) f(s) d s \\
& =f(t+T)+\int_{0}^{t+T} \zeta \exp \{-\underline{\mu}(t+T-s)\} \exp \{\zeta(t+T-s)\} f(s) d s=I I I+
\end{aligned}
$$

where,

$$
\text { IV (29) }
$$

$$
\begin{gathered}
I I I=\int_{-\infty}^{0} \exp \left\{-\int_{s}^{t+T} \mu(\tau, \tau-s, P(\tau)) d \tau\right\} \zeta P(s) d s \\
=\int_{-\infty}^{0} \exp \{-\underline{\mu}(t+T-s)\} \zeta P(s) d s \\
\leq \int_{-\infty}^{0} \zeta P(s) d s[\exp \{-\underline{\mu}(t+T-s)\} \leq 1] \leq \int_{-\infty}^{0} B(s) d s \\
I V=\int_{0}^{t+T} \zeta \exp \{-\underline{\mu}(t+T-s)\} \exp \{\zeta(t+T-s)\} f(s) d s \\
=\int_{0}^{t+T} \zeta \exp \{-\underline{\mu}(t-s)\} \exp \{\zeta(t+T-s)\}\left[\int_{-\infty}^{0} \exp \{-\underline{\mu}(s+T\right. \\
-u)\} \zeta P(u) d u] d s \\
\leq \int_{0}^{t+T} \zeta \exp \{\zeta(t+T-s)\}\left[\int_{-\infty}^{0} \zeta P(u) d u\right] d s \\
\leq \int_{0}^{t+T} \zeta \exp \{\zeta(t+T-s)\}\left[\int_{-\infty}^{0} B(u) d u\right] d s
\end{gathered}
$$

From (29)

$$
P(t+T) \leq \int_{-\infty}^{0} B(s) d s+\int_{0}^{t+T} \zeta \exp \{\zeta(t+T-s)\}\left[\int_{-\infty}^{0} B(u) d u\right] d s
$$

Now,

$|P(t+T)-P(t)|$ 
Nazia Afrin and Md. Shahidul Islam

$$
\begin{aligned}
& \leq \mid \int_{0}^{t+T} \zeta \exp \{\zeta(t+T-s)\}\left[\int_{-\infty}^{0} B(u) d u\right] d s \\
& \quad-\int_{0}^{t} \zeta \exp \{\zeta(t-s)\}\left[\int_{-\infty}^{0} B(u) d u\right] d s \\
& \leq \mid \psi \int_{0}^{t+T} \zeta \exp \{\zeta(t+T-s)\} d s-\psi \int_{0}^{t} \zeta \exp \{\zeta(t-s) d s \mid
\end{aligned}
$$

Taking $\psi=\int_{-\infty}^{0} B(u) d u, \psi>0$.

$$
\begin{gathered}
|P(t+T)-P(t)| \leq|\psi[\exp \{\zeta(t+T)\}-1]+\psi[1-\exp \{\zeta t\}]| \\
\leq|\psi[\exp \{\zeta(t+T)\}-\exp \{\zeta t\}]| \leq|\psi[\{\zeta(t+T)-\zeta t\}]| \leq|\psi[\zeta T]| \leq \epsilon \\
{\left[\psi \leq \frac{\epsilon}{\zeta T}\right]} \\
|P(t+T)-P(t)| \leq \epsilon
\end{gathered}
$$

Similarly, we can prove for $B(t)$.

From this theorem we get unique solution for all time. That is at time $t$ and time $t+T$. Since the solution is unique it means that the solution is same for every time. The solution at time $t$ is $P(t)$ and the solution at time $t+T$ is $P(t+T)$. Now, we can write $P(t+T)=P(t)$, for all $T>0$.

So, we can say that the population model has periodic solution.

\section{Conclusion}

Mathematical models have been developing step by step. Success of modelling of physical or biological phenomena depends on how much appropriately it describes the population in future as well as past. Gurtin-MacCamy model is much more complicated and describes the population sophistically because its parameter depends not only on age and time but also on populations. In this paper, our endeavor was to describe periodic solutions of Gurtin-MacCamy model and extended Gurtin-MacCamy model. We find that these two models have unique solution for some time $t$ and for some time $t+T$, where $T$ is a arbitrary constant and $T>0$, we can also find a unique solution. Difference between these two solutions is very small so they are almost same. Since in time $t$, the model has unique solution so we can say that these two solutions are same. Periodic solution of Gurtin-MacCamy model depends on the total initial population size and periodic solution of Extended Gurtin-MacCamy model depends on the birth rate of the population.

Acknowledgements. We would like to thank the editor and all the anonymous reviewers for their valuable suggestions and comments which improve the presentation of the manuscript.

\section{REFERENCES}

1. J.D.Murray, Mathematical Biology, Third edition, Springer-Verlag, New York, Berlin Heidelberg, (2002).

2. M.N.Islam, Periodic solutions of volterra integral equations, Int. J. Math. Sci., 11(4) (1988) 781-792. 
Periodic Solutions of Gurtin-MacCamy Model and Extended Gurtin-MacCamy Model

3. M.El-doma, Stability analysis for the Gurtin-MacCamy's age-structured population dynamics model, Applications and Applied Mathematics, 2(2) (2007) 144-151.

4. M.N.Islam, Asymptotically periodic solutions of volterra integral equations, Electronic Journal of Differential Equations, 2016 (2016) 1-9.

5. T.A.Burton, T.Furumochi, Periodic and asymptotically periodic solutions of Volterra integral equations, Funkcialaj Ekvacioj, 39 (1996) 87-107.

6. A.D.Polyanin and A.V.Manzhirov, Handbook of Integral Equations, Second Edition, Chapman \& Hall/CRC-Boca Raton London New York, (2008).

7. S.Swarup, Integral Equations, Tenth Edition, (2000).

8. D.R.Smart, Fixed Point Theorems, Cambridge University Press, Cambridge, (1980).

9. C.Corduneanu, Integral Equations and Applications, Cambridge University Press, Cambridge, (1991).

10. M.E.Gurtin and R.C.MacCamy, Non-linear age-dependent population dynamics, Arch. Rat. Mech. Anal., 54 (1974) 281-300.

11. Md. Shahidul Islam and A.F.M. Khodadad Khan, A non-linear population model in the age-time continuum, Dhaka Univ. J. Sci., 45(1) (1997) 47-56.

12. Md. Shahidul Islam, Non-linear age-time population dynamics, Pre Print (2002). 